\title{
ACTIVITY REPORT OF THE IAU WORKING GROUP ON REFERENCE SYSTEMS SUB-GROUP ON ASTRONOMICAL CONSTANTS
}

\author{
T. FUKUSHIMA \\ Satellite Geodesy Office, Geodesy and Geophysics Div., \\ Hydrographic Department, Maritime Safety Agency \\ 5-3-1, Tsukiji, Chuo-ku, Tokyo 104, Japan
}

\section{Introduction}

The IAU Working Group on Reference Systems (WGRS) Sub-Group on Astronomical Constants (SGAC) was established in June, 1989, as a consequence of resolutions adopted by Commissions 4, 7, 8 and 24 at the IAU General Assembly at Baltimore in 1988. The given missions of this sub-group were stated clearly by J. Hughes, the chairman of the WGRS, as:

\begin{abstract}
"Provide numerical values for the primary constants and specify the relationships between these and other, secondary constants within the framework of general relativity. This task will involve the documentation of the constants themselves as well as of the procedures and algorithms associated with their use. Recognition must be given to the fact that approaches which are specific to various techniques exist. The group must recommend the best estimates which can meet the varied requirements of astronomy. The apparent dichotomy between adopting fixed values for various quantities on the one hand, and the nced for current, highly accurate values on the other hand, must be addressed by the group. Indeed, the crafting of effective procedures for incorporating new determinations into the values assigned to the constants, and the setting up of a mechanism for disseminating information regarding new determinations as an interim measure, are important tasks for this group."
\end{abstract}

This is a report of the activity of the SGAC prior to IAU Colloquium No. 127. Section 2 summarizes the questionnaire prepared in the course of discussion. In Section 3, the discussions on major issues are introduced. The drafts of Recommendations from the SGAC prepared before the Colloquium are shown in Section 4. Note that these drafts were very different from the final form as indicated in this Proceeding. Sections 5 and 6 deal with the current status of astronomical constants used widely now and the best estimates of some major constants which are available now, respectively. I believe these will serve as a guideline for current astronomical constants.

\section{Summary of Activity}

After the formation of SGAC, we have discussed the matters of astronomical constants and units through the exchange of letters and about a dozen circulars. Among them, a questionnaire on astronomical constants was sent to all members of WGRS including the SGAC and some other specialists at the beginning of 1990. It contained 22 questions with some possible selections. The essence of the questions are as follows;

1) Should the system of astronomical constants be limited within the solar system?

2) What constants beyond the solar system should be included?

3) What systems of units beyond the solar system are needed?

4) Should standard procedures be included in the scope of the SGAC?

5) How many systems of units should be prepared when taking general relativity into account?

6) If we accept the IAU Recommendation 5 (1976) on time-like arguments, how should we construct the systems of units consistent with it?

7) What combination of defining constants is most appropriate?

8) What expression is most suitable for a primary constant defining the AU? 
9) Should the mean angular velocity of the Earth be included as a primary constant?

10) Should the masses of minor planets and natural satellites except for the Moon be discussed?

11) What changes are needed in the classification of constants?

12) Should accuracies be indicated also?

13) What policy should we take on compatibility with the existing systems of constants in other fields?

14) What new constants should be added?

15) What constants should be excluded from the present system?

16) What mechanism to update the system should be adopted?

17) Is the form of reciprocal masses reasonable?

18) What auxiliary units of length are needed?

19) What auxiliary units of time are needed?

20) Should units of angle be specified?

21) Is there any other question?

22) To whom should we send this questionnaire?

From 22 members and consultants, answers were obtained. They are too long (47 pages) to be quoted here.

\title{
3. Discussed Topics
}

\subsection{RELATIVISTIC EFFECTS ON UNITS}

As stated in the paper of Fukushima et al. (1986), the present IAU convention on time-like arguments to have no secular difference among them will force one to use different sets of units in different coordinate systems within the framework of general relativity; the terrestrial meter and second in the geocentric coordinate system on the one hand, and the barycentric meter and second in the barycentric coordinate system on the other hand, for example. Furthermore both of these units differ from SI units. It was acknowledged that there are two options to solve this problem. The one is to keep the present convention and to introduce different systems of units. In this case, a scaling factor should be introduced to connect different systems of units. The other is to abandon the present convention and to use only one system of units, SI. In any case, it was noted that the numerical values of constants determined by using the TDBbased observation should be examined carefully.

Ref.: Fukushima et al.: 1986, Celestial Mechanics, 36, 215.

\subsection{CHOICE OF DEFINING CONSTANTS}

Kubo argued that

\begin{abstract}
"The defining constants should be the constants such that no inconsistency will be caused by assigning them whatever numerical values. The Gaussian constant $k$ defines the length of AU. However, we should remark that $k$ never fixes the length of $\mathrm{AU}$. If the physical magnitude of $G M_{\text {Sun }}$ changes, the length of $\mathrm{AU}$ will change even if $k$ is kept the same. The length of AU should be unchanged even the mass of actual Sun or $G$ will change, shouldn't it? To define AU by means of $k$ is just the same as to define a day by means of the mean solar day, isn't it? If so, we must define $\mathrm{AU}$ by the light time for unit distance $\tau_{\mathrm{A}}$ and we should adopt $\tau_{\mathrm{A}}$ as a defining constant."
\end{abstract}

Some members supported his idea, however many others preferred to keep the present style, namely $k$ and $c$ as defining constants.

\subsection{DISCRIMINATION OF CONSTANTS AND QUANTITIES}

The answers to the questionnaire indicated the tendency to extend the coverage of the system of astronomical constants to include, for example, the transformation matrix between FK5 and a galactic reference frame, the 
mean rotational angular velocity of the Earth, $G M$ of many natural satellites and so on. However, it is clear that the degree of accuracy for estimated numerical values differs very much depending on the nature of the determination. Some constants have more than 10 well-determined digits while others are estimated with $50 \%$ accuracy. Also it was argued that there are two contradictory requirements on constants; 1 ) to seek the latest and most accurate values and to update them as frequently as possible, and 2) to keep them as standards for long-term references. To solve this dilemma, Sinclair proposed to discriminate primary and secondary constants and to call the latter as "best estimates". This proposal was welcomed by many members, however, to draw a line between them will require a deep consideration as Seidelmann stressed. Also many people felt that to extend the coverage is beyond the mission of the SGAC even if it is desirable.

\subsection{NEED FOR STANDARD PROCEDURES}

Almost all replies stressed the importance of providing standard procedures in fundamental astronomy. In other words, they required a kind of IAU version of the IERS Standards. The IERS Standards do not cover the whole of fundamental astronomy so that another standards, say IAU Standard Procedures, are required. Also possible media for their distribution were discussed; to utilize E-mail systems or to provide them in a machine-readable form. However, some members argued that to prepare them is far beyond the mission of the SGAC. So it was proposed to establish a special working group for their establishment.

\subsection{UPDATE MECHANISM}

The update mechanism of the geodetic system of constants and their best estimates in the IAG was discussed and almost all members and consultants agreed to introduce a similar kind of mechanism into the IAU. Namely, to keep a system of constants for long-term references and to update a list of best estimates for other specified 'quantities' at every General Assembly.

\section{Proposed Recommendations}

Note that the proposed Recommendations listed in the following are different from the final ones. They are quoted here just to show the change of opinions in the course of discussions in our sub-group.

Recommendation C1: Separation of constants and quantities in astronomy and the establishment of a permanent working group to maintain the list of astronomical quantities

recognizing the importance in astronomy to discriminate the well-cstablished constants and the quantitics whose estimates will be improved frequently,

recommends that the former constants and the units remain as a system of astronomical units and constants which should be used as numerical standards to produce long-term references, and should be unchanged unless the adopted values of constants deviate greatly from their latest estimates or the structure of system becomes inadequate due to the increased knowlcdge,

that the estimates of latter quantities shall be presented and updated at every General Assembly from now on, and

that a permanent working group named the Working Group on Astronomical Quantities (WGAQ) shall be established for the update and improvement of the list of such estimates.

\section{Recommendation C2: IAU (1991) System of Astronomical Units and Constants}

recommends that the attached list of units and constants Annex I (dropped from this report) shall be adopted as the "IAU (1991) System of Astronomical Units and Constants", which should be used as the numerical standards to produce long-term references in astronomy.

Recommendation C3: IAU (1991) Estimates of Astronomical Quantities 
recommends that the attached list of estimates of quantities Annex II (dropped from this report) shall be referred as the "IAU (1991) Estimates of Astronomical Quantities", which can be used as numerical standards in astronomy.

Recommendation C4: Establishment of electronic accesses to the IAU (1991) System of Astronomical Units and Constants and the IAU (1991) Estimates of Astronomical Quantities

noting that the recommended lists of constants and estimates of quantities contain a great deal of digits in their expressions,

recognizing the necessity to avoid mistakes caused by copying these digits by hands,

recommends establishing electronic accesses to these lists.

Recommendation C5: Establishment of a working group to prepare the IAU Standards of Procedures

noting that the MERIT Standards and the IERS Standards have contributed significantly in the progress of astronomy and geodesy,

that these standards of procedures do not cover the whole of basic astronomy, and

recognizing that it is not sufficient for the establishment of references to prepare only the numerical standards, namely a system of astronomical constants and a list of estimates of astronomical quantities, unless the standard procedures for using these numerical values are also given,

recommends establishing a working group named the Working Group on Standards of Procedures (WGSP) to prepare a draft report on standards of procedures needed in astronomy, which

1) should have a maximum degree of compatibility with the IERS Standards,

2) should include the implementations of procedures in the form of tested software as often as possible, and

3) should be made available not only in the form of literature but also in the form which computers can read easily,

at least six months before the next General Assembly.

\section{Current Systems of Astronomical Constants}

There are three sets of astronomical constants currently used as self-consistent systems; the IAU 1976 System, DE200 System and IERS Standards (1989). Note that both the Connaisance des Temps (French Ephemeris) and the Japanese Ephemeris were obtained by fitting their positional values to those of DE200 but by using the IAU1976 System. While the Astronomical Almanac and other national ephemerides are based on the DE200 both in positional values and in the system of constants. Some constants are the same for these three systems;

$\begin{array}{llcl}k & = & 0.01720209895 & \mathrm{AU}^{3 / 2} \mathrm{day}^{-1} M_{\text {Sun }}{ }^{-1 / 2}, \\ c & = & 299792458 & \mathrm{~m} / \mathrm{s}, \\ p & =\quad 5029.0966 & \mathrm{n} / \mathrm{jc}, \\ M_{\text {Sun }} / M_{\text {Mercury }} & = & 6023600, & \\ M_{\text {Sun }} / M_{\text {Venus }} & = & 408523.5, & \\ M_{\text {Sun }} / M_{\text {Mars }} & = & 3098710, & \\ M_{\text {Sun }} / M_{\text {Neptune }} & = & 19314, & \end{array}$

where square brackets denote a derived constant. Constants whose values are different among three systems are shown in the table below where the units for $G, G M_{\text {Earth }}$ and $G M S_{\text {un }}$ are $10^{-11} \mathrm{~m}^{3} /\left(\mathrm{kg}^{*} \mathrm{~s}^{2}\right)$, 
$10^{+14} \mathrm{~m}^{3} / \mathrm{s}^{2}$ and $10^{+20} \mathrm{~m}^{3} / \mathrm{s}^{2}$, respectively. In the table, dashes mean that the value of corresponding constant is not defined explicitly in the literature nor able to be computed from other defined values in the system.

\begin{tabular}{lccc}
\hline Constants & IAU1976 & DE200 & IERS1989 \\
\hline$\tau_{\mathrm{A}} / \mathrm{s}$ & 499.004782 & {$[499.00478370]$} & 499.00478370 \\
$\mathrm{AU} / \mathrm{m}$ & {$[149597870]$} & 149597870.66 & {$[149597870.66]$} \\
$G$ & 6.672 & - & 6.67259 \\
$a_{e \text { Earth }} / \mathrm{m}$ & 6378140 & - & 6378136 \\
$J_{2 \text { Earth }}$ & 0.00108263 & - & 0.001082626 \\
$G M_{\text {Earth }}$ & 3.986005 & {$[3.98600448]$} & 3.98600448 \\
$f_{\text {Earth }}$ & {$[1 / 298.257]$} & - & {$[1 / 298.257]$} \\
$\left.M_{\text {Sun }} / M_{\text {Earth }}+M_{\text {Moon }}\right)$ & {$[328900.5]$} & 328900.55 & {$[328900.55]$} \\
$M_{\text {Sun }} / M_{\text {Earth }}$ & {$[332946.0]$} & {$[332946.038]$} & DE200 \\
$M_{\text {Moon }} / M_{\text {Earth }}$ & 0.01230002 & {$[0.012300034]$} & 0.012300034 \\
$M_{\text {Earth }} M_{\text {Moon }}$ & {$[81.30068]$} & 81.300587 & {$[81.300588]$} \\
$\varepsilon_{\mathrm{o}}$ & $23^{\circ} 26^{\prime} 21^{\prime \prime} .448$ & {$\left[23^{\circ} 26^{\prime} 21^{\prime \prime} .4119\right]$} & DE200 \\
$M_{\text {Sun }} / M_{\text {Jupiter }}$ & 1047.355 & 1047.350 & $D E 200$ \\
$M_{\text {Sun }} / M_{\text {Saturn }}$ & 3498.5 & 3498.0 & DE200 \\
$M_{\text {Sun }} / M_{\text {Uranus }}$ & 22869 & 22960 & DE200 \\
$M_{\text {Sun }} / M_{\text {Pluto }}$ & 3000000 & 130000000 & DE200 \\
$\pi_{\text {Sun }}$ & {$\left[8^{\prime \prime} .794148\right]$} & - & {$\left[8^{\prime \prime} .794141\right]$} \\
$G M_{\text {Sun }}$ & {$[1.32712438]$} & {$[1.327124399]$} & {$[1.32712440]$} \\
\hline
\end{tabular}

Ref.: (IAU1976) Duncombe et al:: 1977, Transactions of the IAU, XVIB, 56.

(DE200) Standish: 1990, Astron. and Astrophys., 223, 252.

(IERS1989) McCarthy et al.: 1989, IERS Tech. Note, No.3, 1.

This table clearly shows that the numerical standards in the IERS Standards (1989) were mostly based on the DE200 System though its classification of primary and derived constants differs from that of the DE200 System sometimes. Note that the listed values of $f$ Earth are different with each other though they seem to be the same. This is because $f_{\text {Earth }}$ is analytically derived from the values of $a_{e}$ Earth,$J_{2 \text { Earth }}$ and $G M_{\text {Earth }}$ plus the mean angular velocity of the Earth rotation $\omega_{E a r t h}$, and the adopted values of first three are different in the IAU1976 System and in the IERS Standards (1989). The adopted values of $\omega_{\text {Earth }}$ are the same in these two systems and is

$$
\omega_{\text {Earth }}=7.292115 \times 10^{-5} \mathrm{radian} / \mathrm{s}
$$

The readers can consult with the explanation of the Geodetic Reference System 1980 (GRS1980) by Moritz for the further information on the derivation procedures of geodetic constants such as $f$ Earth.

Ref.: $\quad$ (GRS1980) $\quad$ Moritz: 1988, Bulletin Géodésique, 62, No. 3, 348.

\section{Current Best Estimates}

The followings are the best estimates of astronomical constants whose references are available at present, i.e. October, 1990. We must note that some of these are not final determinations and research to update these values is still on going. Also note that these estimates were all derived by using TDB as a time-like argument in the solar system barycentric coordinate system. Thus, if we adopt the way to allow secular 
differences among the time-like arguments, the numerical value of some constants shall be changed, especially for those with more than 8 significant digits.

WARNING: Much care should be taken in the use of following estimated values in conjunction with ephemerides or star catalogs which are based on the existing systems of astronomical constants such as described in the preceding section!

\subsection{LIGHT TIME FOR AU}

Best estimates of $\tau_{\mathrm{A}}$ and/or $\mathrm{AU} / \mathrm{m}$ have been obtained as a solve-for parameter in creating planetary ephemerides. The following is a table of their values determined in creating JPL's DE series.

\begin{tabular}{|c|c|c|}
\hline System/Ephemerides & $\mathrm{AU} / \mathrm{m}$ & $\tau_{\mathrm{A}} / \mathrm{s}$ \\
\hline IAU1976 & [149597870] & 499.004782 \\
\hline DE96 & 149597871411 & [499.006708] \\
\hline DE102 & 0683 & 3779] \\
\hline DE108 & 0705 & 3853] \\
\hline DE111 & 0652 & $3676]$ \\
\hline DE118, DE200 & 0660 & 3703] \\
\hline DE125, DE201 & 0614 & $3549]$ \\
\hline DE130, DE202 & 0609 & 3533] \\
\hline
\end{tabular}

Ref.: (DE96-DE118, DE200)

Standish: 1990, Astron. and Astrophys., 223, 252.

(DE125, 130; DE201, 202) Standish: 1990, private communication.

Standish reported that the standard deviation for AU/m is \pm 50 for the DE118 or DE200 value because $A U / m$ would change by that amount as a result of improvement of asteroid modelling. He also said that the DE130/DE202 was not a final version based on the latest observations including Voyager encounters with outer planets. Therefore, we do not recommend any value of these as a best estimate at present.

\subsection{PRECESSION CONSTANT}

According to McCarthy, there have been the following estimates of a correction to the precession constant in IAU1976 System, where the unit is mas/Julian year $=0 " .1 /$ jc.

$\begin{array}{lll}-2.39 & ( \pm & 0.13) \\ -5.00 & ( \pm & 1.10) \\ -1.80 & ( \pm & 0.13) \\ -2.05 & ( \pm & 0.15) \\ -3.76 & ( \pm & 0.47) \\ -2.53 & ( \pm & 0.24) \\ -2.22 & ( \pm & 0.14) \\ -2.7 & ( \pm & 0.4)\end{array}$

Herring et al:: 1986, Jour. Geophys. Res., 91, 4745.

Herring: 1988, BIH Annual Report for 1987, D-106.

However, Herring recommended the correction -3.00 .

Sovers and Edwards: 1988, BIH Annual Report for 1987, D-109.

Steppe et al.: 1989, IERS Tech. Note, No.2.

Zhu et al:: 1989, Astron. Jour. 99, 1024.

McCarthy and Luzum: 1990, to be submitted to Astron. Jour.

Whipple: 1990 , private communication.

Williams et al.: 1990, Submitted to Astron. and Astrophys. Let.

This paper gives also an estimate of general precession as $5028^{\prime \prime} .82 / \mathrm{jc}$.

We take the unweighted mean of these except for the value -5.00 . The resulting correction becomes -2.493 $( \pm 0.634)$. After rounding the last digit, we have a best estimate of the precession constant as

$$
p=5028.847( \pm 0.063) " / j \mathrm{c}
$$


However, we should remark that this value is not compatible with the published national ephemerides, the existing star catalogs such as FK5, nor the IAU (1976) Precession theory.

\subsection{OBLIQUITY OF THE ECLIPTIC}

According to Standish, best estimates of the obliquity of ecliptic have been obtained dynamically from the analysis of motion of ecliptic realized in the planetary ephemerides. In this sense, it is not a primary constant but a kind of derived constant although its derivation procedure is complicated. The following is a table of their values determined from JPL's DE series and an analytical planetary theory VSOP82.

\begin{tabular}{lr}
\hline System/Ephemerides & $\varepsilon_{0}$ \\
\hline IAU1976 & $23^{\circ} 26^{\prime}$ \\
DE96 & .448 \\
DE102 & .327 \\
DE108 & .412 \\
DE111 & .310 \\
DE118, DE200 & .412 \\
VSOP82 & .412 \\
DE125, 130; DE201, 202 & .409 \\
\hline
\end{tabular}
Ref.: (DE96-DE1 18, DE200) Standish: 1990, Astron. and Astrophys., 223, 252. (VSOP82) Bretagnon: 1982, Astron. and Astrophys., 114, 278. (DE125, 130; DE201, 202) Standish: 1990, private communication.

Judging from the trend of convergence seen in this list, we recommend its best (derived) estimate as

$$
\varepsilon_{0}=23^{\circ} 26^{\prime} 21^{\prime \prime} .411( \pm 0 " .002) \text {. }
$$

However, we should remark that this value is not compatible with the published national ephemerides, the existing star catalogs such as FK5, nor the IAU (1976) Precession theory. Also note that one should use the corresponding value of obliquity when one uses a certain planetary ephemeris.

\subsection{EARTH-MOON MASS RATIO}

Best estimates of the Earth-Moon mass ratio have been obtained as a solve-for parameter in creating planetary ephemerides. The following is a table of their values determined and/or adopted in JPL's DE series. This value may be better treated as a derived constant to be derived from the two primary constants $G M_{\text {Earth }}$ and $M_{\text {Sun }} / M_{\text {Earth }}+$ Moon with the use of $\tau_{\mathrm{A}}$ or $\mathrm{AU} / \mathrm{m}$.

\begin{tabular}{|c|c|c|}
\hline System/Ephemerides & $M_{\text {Earth }} / M_{\text {Moon }}$ & $\mu=M_{\text {Moon }} / M_{\text {Earth }}$ \\
\hline IAU1976 & [81.300681] & 0.01230002 \\
\hline DE96, 102 & 81.3007 & {$[0.0123000171]$} \\
\hline DE108 & 49 & {$\left[\begin{array}{ll}{[} & 489\end{array}\right]$} \\
\hline DE111, 118-130; DE200-202 & 587 & 342] \\
\hline
\end{tabular}

Ref.: (DE96-DE118, DE200) Standish, 1990: Astron. and Astrophys., 223, 252. (DE125, 130; DE201, 202) Standish, 1990: private communication. 
According to Standish, the values in the table below are the current best estimates of $G M$ of the planets, where square bracket denotes the derived value using the DE200 estimate of $A U / m=149597870660$. Note that these $G M$ Planet values include the contribution of satellites.

\begin{tabular}{lclll}
\hline Planet & \multicolumn{3}{c}{$M_{\text {Sun }} / M_{\text {Planet }}$} & $G M$ Planed $\left(\mathrm{km}^{3} / \mathrm{s}^{2}\right)$ \\
\hline Mercury & 6023600. & $( \pm 250)$ & & \\
Venus & {$[408523.71$} & $( \pm 0.06)]$ & 324858.60 & $( \pm 0.05)$ \\
Earth+Moon & 328900.55 & & & \\
Mars & 3098708. & $( \pm 0)$ & & \\
Jupiter & {$[1047.3486$} & $( \pm 0.0008)]$ & 126712767. & $( \pm 100)$ \\
Satum & 3497.898 & $( \pm 0.018)$ & & \\
Uranus & 22902.94 & $( \pm 0.04)$ & & \\
Neptune & {$[19412.240$} & $( \pm 0.057)]$ & 6836534. & $( \pm 20)$ \\
Pluto+Charon & 1.350 & $( \pm 0.006) \times 10^{+8}$ & & \\
\hline
\end{tabular}

Ref.: (Mercury)

Anderson et al.: 1987, Icarus, 71, 337.

(Venus)

This is the same as those in IAU1976, DE200 and IERS1989 systems.

(Earth+Moon) Standish: 1987, "Ephemerides DE130/LE130 \& DE202/LE202", JPL Interoffice Memo., 314.6-891.

This is the same as that in DE20

(Mars) Null: 1969, Astron. Jour., 72, 1292.

(Jupiter) Campbell and Synnott: 1985, Astron. Jour., 90, 364.

(Satum) Campbell and Anderson: 1989, Astron. Jour., 97, 1485.

(Uranus) Anderson et al.: 1987, Jour. Geophys. Res., 92, 14877.

(Neptune) Tyler et al.: 1989, Science, 246, 1466.

This paper gives a value for the reciprocal ratio to $G M_{S}$ un, however, the conversion was done using an old value for the speed of light. A correct value is shown here.

(Pluto+Charon) Derived from Tholen and Buie: 1988, Astron. Jour., 96, 1977.

The value and uncertainty are derived from the cited values of $19640( \pm 320) \mathrm{km}$ for the semi-major axis and $6.387230( \pm 0.000021)$ days for the period of the orbital motion of Pluto+Charon.

\section{Acknowledgment}

This report is a product of the cooperative work of not only members of the SGAC but also other members of the WGRS and some distinguished experts, whose names are listed in the Appendix. Here I would like to thank all those who returned answers to the questionnaire, participated in the discussion, sent fruitful comments and information, and prepared some parts of this report. 
Appendix. Members and Consultants of SG on Astronomical Constants

\section{MEMBERS}
M. Bursa,
Astronomical Institute, Praha, Czechoslovakia.
J. Campbell, Geodătisches Institut der Univ. Bonn, F.R. Germany.
J. Chapront, Bureau des Longitudes, Paris, France.
W.-J. Jin, Shanghai Observatory, Shanghai, P.R. China.
G. Krasinsky, Institute of Applied Astronomy, Leningrad, U.S.S.R. R.D. Reasenberg, Center for Astrophysics, Cambridge, U.S.A.
P.K. Seidelmann, U.S. Naval Observatory, Washington, D.C., U.S.A.
A.T. Sinclair, Royal Greenwich Observatory, Cambridge, U.K.
E.M. Standish, Jr., Jet Propulsion Laboratory, Pasadena, U.S.A.
Ch. Veillet, $\quad$ C.E.R.G.A., Grasse, France.

\section{CONSULTANTS}
S. Aoki,
C. Boucher,
V.A. Brumberg,
Z.-G. Cheng,
V. Dehant,
E. Groten,
B. Guinot,
Y. Kubo,
National Astronomical Observatory, Tokyo, Japan.
Institut Géographique National, Saint-Mandé, France.
D.D. McCarthy,
C.A. Murray,
M. Sasaki,
Institute of Applied Astronomy, Leningrad, U.S.S.R.
Shanghai Observatory, Shanghai, P.R. China.
Observatoire Royal de Belgique, Bruxelles, Belgique.
Institut für Phys. Geodäsie, Darmstadt, F.R. Germany.
C.A. Smith, Jr.,
B.I.P.M., Sèvres Cedex, France.
Hydrographic Department, Tokyo, Japan.
U.S. Naval Observatory, Washington, D.C., U.S.A.
Eastbourne, Sussex, U.K.
Hydrographic Department, Tokyo, Japan.
M.H. Soffel,
M. Sôma,
U.S. Naval Observatory, Washington, D.C., U.S.A.
R.O. Vicente,
Univ. of Tübingen, F.R. Germany.
National Astronomical Observatory, Tokyo, Japan.
S.-X. Wu,
B.-X. Xu, Lisboa, Portugal.
Xian Branch of Academia Sinica, Shaanxi, P.R. China.
T. Yoshino, Nanjing Univ., Nanjing, P.R. China.
Communication Research Labolatory, Tokyo, Japan. 